\title{
A Handy Primer for Medical Educators
}

\section{The College Classroom Assessment Compendium: A Practical Guide to the College Instructor's Daily Assessment. By Jay Parkes and Dawn Zimmaro; Routledge; New York; 2018; ISBN: 978-1- 138-24026-7; pp. 218; \$31.96 (paperback).}

\author{
Mary Morreale ${ }^{1}$ \\ Received: 3 July 2018 / Accepted: 10 July 2018 / Published online: 1 August 2018 \\ (C) Academic Psychiatry 2018
}

When I was appointed to a role in medical student teaching years ago, I quickly became concerned that I had never received any formal education on how to teach. I read what I could, attended conferences, and looked for help from seasoned mentors, but still felt out on a limb. What type of assessments should I choose? Should students be allowed to work for extra credit? How should I handle late assignments? The College Classroom Assessment Compendium: A Practical Guide to the College Instructor's Daily Assessment would have been a great help to me then, particularly when I was tasked with writing a syllabus and developing fair and consistent educational policies, because it concretely and concisely addresses many fundamentals of teaching.

Although the book is written for a broader audience than simply medical educators, the vast majority of the book is pertinent to those in our profession. The authors discuss multiple topics relevant to the administration of preclinical courses, such as aligning assessments to learning objectives, selecting types of grading schemes, dropping questions from examinations, and ensuring test security. For those who are clerkship directors, the chapters on feedback, summative assessments, in-class participation, and utilizing rubrics to foster more objective grading strategies are among those that are applicable. This book addresses larger issues as well, for example, humor in the classroom, academic beneficence, and evaluation anxiety.

One of the most important things I learned after reading this book is how important it is to create well thought-out policies

Mary Morreale

mmorreale@med.wayne.edu

1 Wayne State University, Detroit, MI, USA before any teaching activity is undertaken. It is much easier to approach matters like cheating and plagiarism, missed assignments, and borderline grade cases when one has the "opportunity to think thoroughly and dispassionately about the issues at hand" (p. 167). The College Classroom Assessment Compendium: A Practical Guide to the College Instructor's Daily Assessment would be an excellent place to start when thinking about appropriate educational practices and policies because it covers a broad array of topics in practical and concise terms.

The format of this book (59 chapters that range between 2 and 6 pages) allows for easy readability and reference. The authors created it to provide "just-in-time learning" (p. 2). For example, if a student misses a required activity or fails to turn in an assessment on time, the reader can quickly read the entries on "Attendance," "Missing Assignments," and "Late Work" before acting. The topics in the book are organized alphabetically, and both the table of contents and the index make finding what one needs quite simple. Each entry contains references to more in-depth discussions, as well as a "see also" list, which identifies similar topics within the book.

In sum, The College Classroom Assessment Compendium: A Practical Guide to the College Instructor's Daily Assessment is a vital addition to the bookshelf of any teacher who is in the process of developing or refining a curriculum. Written by experts in educational psychology, learning design, and assessment, this book just might be the missing piece for those of us seeking more education on how to educate others!

\section{Compliance with Ethical Standards}

Disclosures The author states that there is no conflict of interest. 